\title{
Co-design of the LCL Filter and Control for Grid-Connected Inverters
}

\author{
Yu Zhang ${ }^{\dagger}$, Mingyu Xue*, Minying Li ${ }^{* *}$, Yong Kang*, and Josep M. Guerrero ${ }^{* * *}$ \\ ${ }^{\dagger *}$ State Key Laboratory of Advanced Electromagnetic Engineering and Technology (AEET), Huazhong University of \\ Science and Technology, Wuhan, China \\ ${ }^{* *}$ Guangdong Zhicheng Champion Group Company, Dongguan, China \\ *** Department of Energy Technology, Aalborg University, Aalborg, Denmark
}

\begin{abstract}
In most grid-connected inverters (GCI) with an LCL filter, since the design of both the LCL filter and the controller is done separately, considerable tuning efforts have to be exerted when compared to inverters using an L filter. Consequently, an integrated co-design of the filter and the controller for an LCL-type GCI is proposed in this paper. The control strategy includes only a PI current controller and a proportional grid voltage feed-forward controller. The capacitor is removed from the LCL filer and the design procedure starts from an L-type GCI with a PI current controller. After the PI controller has been settled, the capacitor is added back to the filter. Hence, it introduces a resonance frequency, which is identified based on the crossover frequencies to accommodate the preset PI controller. Using the proposed co-design method, harmonic standards are satisfied and other practical constraints are met. Furthermore, the grid voltage feed-forward control can bring an inherent damping characteristic. In such a way, the good control performance offered by the original L-type GCI and the sharp harmonic attenuation offered by the latter designed LCL filter can be well integrated. Moreover, only the grid current and grid voltage are sensed. Simulation and experimental results verify the feasibility of the proposed design methodology.
\end{abstract}

Key words: Co-design, Current control, Grid-connected inverter, Grid voltage feed-forward control, LCL filter, Resonance damping

\section{INTRODUCTION}

Grid-connected inverters (GCIs) are widely used in distributed generation (DG) systems where the sources are mostly renewable energies like wind, solar, etc. The traditional L filter is gradually being replaced by LCL filters, due to their stronger harmonic elimination capability [1], [2].

A PI controller is a simple robust controller that can be effectively used for current control in GCIs with an L filter. This is due to the fact that PI controller are easy to design to achieve good performance. For instance, in [3], a PI controller was designed to achieve a maximum crossover frequency while considering the control delay and the PWM

Manuscript received Mar. 26, 2014; accepted Jun. 8, 2014

Recommended for publication by Associate Editor Sung-Yeul Park.

${ }^{\dagger}$ Corresponding Author: zyu1126@mail.hust.edu.cn

Tel: +86-2787543658-810, Fax: +86-2787543658-816, HUST

* State Key Laboratory of Advanced Electromagnetic Engineering and

Technology (AEET), Huazhong Univ. of Science and Tech., China

${ }^{* *}$ Guangdong Zhicheng Champion Group Company, China

*** Department of Energy Technology, Aalborg University, Denmark transport delay. When combined with proportional grid voltage feed-forward control, grid disturbances can also be rejected effectively.

However, the design of PI controller for an LCL-type GCI is not easy due to the resonance of the LCL filter, which may lead to instability and a complex design in terms of the grid current feed-back controllers and grid voltage feed-forward controllers [4]-[6]. With the conventional design method, using PI current control is not enough to achieve stability. In [7], the regions of the active damping control decided by the resonance frequency were discussed. It was concluded that active damping was needed for lower resonance frequencies. Most of the proposed active damping strategies are realized by using multi-variable feedback controls [8]-[12]. Some of them may also lower the system order by using multi-variable feedback controls [13]-[15]. However, multi-variable feedback control lifts the cost of the sensors and makes the control strategy more complicated. Therefore, in [4], a filter-based active damping solution is proposed. In [16], it is concluded that when converter-side current is used as a 
control feedback variable, there is an inherent damping term embedded in the control loop, which can neutralize the resonance introduced by an LCL-filter. This damping term can also be obtained when grid feed-forward is implemented, which will be discussed in this paper.

When the resonance frequency increases, the phenomenon of the magnitude curve crossing over $0 \mathrm{~dB}$ three times can be observed in the Bode diagram of an LCL-type GCI with a PI controller. Hence, according to the Nyquist Stability Criterion [17], three crossover frequencies should be considered together to confirm the stability. However, this has not been discussed in the literature. To accurately ensure stability, three cross over frequencies will be discussed in this paper. They are considered together in the design according to the Nyquist Stability Criterion.

In the conventional designs of an LCL-type GCI, the design of both the LCL filter and the controller is done separately. They always start with the parameter design of the LCL filter, which aims to satisfy switching current harmonic standards and other practical constraints [18]-[20]. Thereafter, the controller is added and designed according to this preset LCL filter. For the LCL filter, various optimization goals are pursued, such as minimum reactive power [21], low volume, low loss [22], and highest power efficiency [23]. In some converters, the controller is considered during the design of the filter. In [24], a generalized design method of an LCL-type active power filter is proposed, with a capacitor current feedback control loop being used for active damping. In [25], the LC filter of the inverter is designed when considering the performance of the close-loop system. However, among the conventional methods of GCI design, most of the power stage filters and controllers are designed separately. Hence, LCL-type GCIs suffer from complexity in terms of hardware and software, and are hard to properly tune. Sometimes poor performance is achieved in comparison to the L-type GCIs.

In DG systems, the primary task of energy conversion requires a further simplification such as a lower number of variables to be sensed. As required by industry applications, GCIs should behave well in terms of current reference tracking, grid disturbance rejecting, and robustness to grid impedance variations. All of these are well featured by L-type GCIs. Hence, a novel co-design method for an LCL-type GCI and its controller is proposed in this paper to achieve all of these purposes. This is theoretically feasible based on multi crossover frequencies according to the Nyquist Stability Criterion.

In the first step of the co-design method proposed in this paper, the capacitor is removed from the LCL filter. This converts it to an L type filter, so that the design starts from an L-type GCI employing a PI controller. After the PI controller has been set for the $\mathrm{L}$ filter, the capacitor is added back to the $\mathrm{L}$ filter. This will introduce a resonance frequency to the LCL filter, which will be adapted to the preset PI controller by identifying the phase margin of three crossover frequencies. Furthermore, proportional feed-forward control of the grid voltage is employed to ensure performance under a highly distorted or weak grid. This brings an inherent damping characteristic. By an easy method, the LCL filter and controller can be co-designed, and both good close-loop performance and sharp harmonic attenuation can be achieved. Moreover, only the output current and grid voltage are sensed. Simulation and experiment results verify the feasibility of the proposed co-design method.

\section{CONCEPT OF THE PROPOSED CO-DESIGN}

The power stage model and controller of a GCI with an LCL filter is shown in Fig. 1(a), where the dc link can be simplified as a constant voltage $U_{d c}$ if the dc capacitor $C_{d}$ is large enough. Resistors $r_{1}, r_{2}$ and $r_{\mathrm{g}}$ can be neglected, while the grid internal impedance $L_{g}$ is considered [16]. The corresponding control scheme is presented in Fig. 1(b), where $G_{P I}(s)$ is the PI current controller and $G_{f f}(s)$ is the grid voltage feed-forward controller.

When the capacitor $C_{f}$ is removed from the LCL filter, the filter becomes an L type, and $L=L_{1}+L_{2}$. In Fig. 1(b), this means to "short-circuit" the dashed block in the power stage model. The $e^{-s T_{d}}$ term represents the system delay, and $T_{d}$ is the one-sample control delay plus the half-sample PWM transport delay [3].

For an L-type GCI, a PI current controller together with a unity proportional feed-forward controller for the grid voltage is commonly employed for current reference tracking and grid voltage disturbance rejection. The state of the art technique for designing a PI current controller is based on maximizing the proportional part and minimizing the integral time constant to obtain the maximum crossover frequency. This results in a good coordination between the dynamics and the steady-state.

In detail, a PI controller $G_{P I}(s)=k_{P}(1+1 / \tau s)$ for an L-type GCI is governed by: $k_{P}=\omega_{c(\max )} L / U_{d c}$ and $\tau=10 / \omega_{c(\max )}$, where $\omega_{c(\max )}$ represents the maximum cross over frequency that determines the expected phase margin $\phi_{m}$ [3].

As a result of this design, the open-loop transfer function from the duty-cycle $d$ to the output-current $i_{2}$ is shown as:

$$
G_{L}^{o}(s)=\frac{i_{2}(s)}{d(s)}=\frac{U_{d c} G_{P I}(s) e^{-s \mathrm{~T}_{\mathrm{d}}}}{\left(L_{1}+L_{2}\right) s}=\omega_{c(\max )}\left(1+\frac{\omega_{c(\max )}}{10 s}\right) \frac{e^{-s \mathrm{~T}_{\mathrm{d}}}}{s}(1)
$$

Fig. 2(a) shows an open-loop Nyquist diagram of an L-type GCI after the design. GCIs with an L filter have a low 


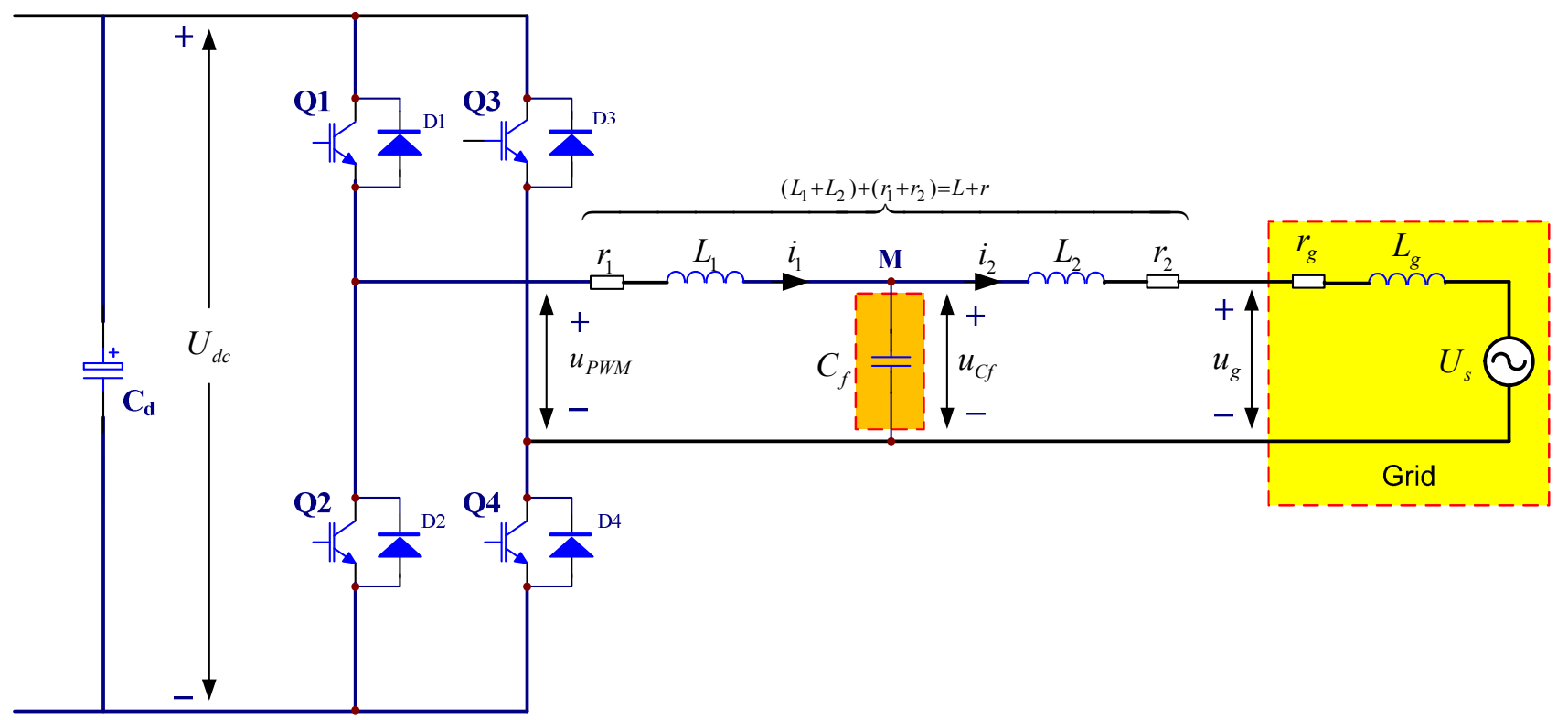

(a) Topology.

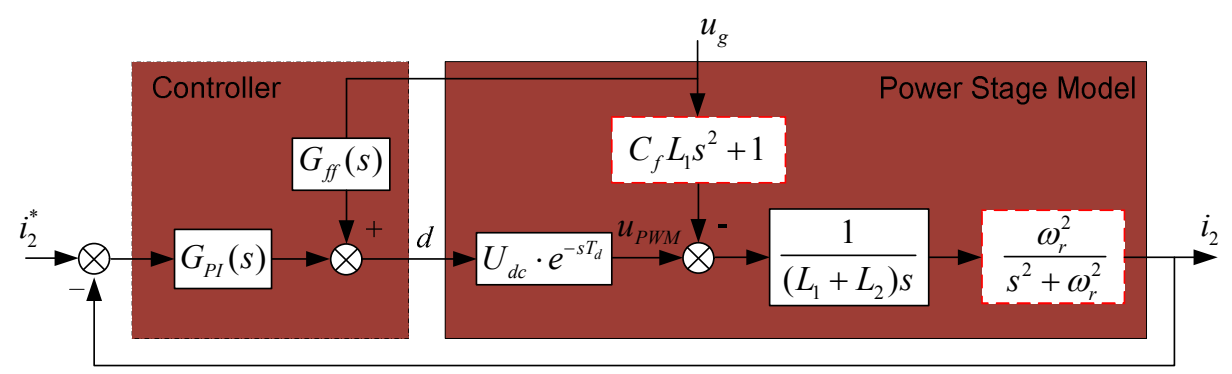

(b) Control models.

Fig. 1. Single-phase GCI with LCL filter.

harmonic attenuation capability. Hence, they can be improved by an LCL filter, which can be realized by returning the filter capacitor $C_{f}$ to the splitting point $\mathrm{M}$ of the $\mathrm{L}$ filter, as shown by the dashed block in Fig. 1(a). For the LCL structure, the open-loop transfer function from the duty-cycle to the output-current is given by:

$$
G_{L C L}^{o}(s)=\frac{i_{2}(s)}{d(s)}=\frac{U_{d c} G_{P I}(s) e^{-s T_{\mathrm{d}}}}{L_{1} L_{2} C_{f} s} \frac{1}{s^{2}+\omega_{r}{ }^{2}}=G_{L}^{o}(s) \frac{\omega_{r}{ }^{2}}{s^{2}+\omega_{r}{ }^{2}}
$$

here, $\omega_{r}=\sqrt{\left(L_{1}+L_{2}\right) / L_{1} L_{2} C_{f}}$ is the resonance frequency of the LCL filter.

With the PI controller $G_{P I}(s)$ unchanged and $G_{L}^{o}(s)$ remaining invariant with a total inductance $L=L_{1}+L_{2}$, the stability after upgrading relies on the value of the resonance frequency $\omega_{r}$. Based on (2), Fig. 2(b)-(c) illustrates the open loop Nyquist diagrams of LCL-type GCIs with three different filter resonance frequencies, which are represented in p.u. values, with the sampling frequency $1 / T$ as the base frequency, i.e. $\omega_{r, p u}=\omega_{r} \mathrm{~T}$. These Nyquist curves show the loci determined by a positive $\omega$ from 0 to infinity. The radius of the semicircles in (b)-(d) is infinity, which is the result of the filter resonance frequency. $P_{\text {crit }}$ denotes the critical point: $-1+\mathrm{j} 0$.

It can be estimated from (2) that the Nyquist curve will cross the negative real axis from the left of the critical point $\mathrm{P}_{\text {crit }}$ when $\omega_{r, p u}<1.06$, which means instability. However, it will cross the negative real axis from the right of $\mathrm{P}_{\text {crit }}$ when $\omega_{r, p u}>1.06$, which means stability. Furthermore, if $\omega_{r, p u}$ is increased to 9 , the GCI will return to instability. Therefore, the determination of $\omega_{r, p u}$ is very important in the design.

It is convenient to further discuss the relative stability in the Bode diagram. Fig. 3 illustrates a Bode diagram through three different filter resonance frequencies (see lines 1, 2, 3).

It can be seen that in the case of a lower $\omega_{r, p u}=1$ (see line 1 ), there is only one negative crossing of $0 \mathrm{~dB}$ in the whole frequency range. In addition, the phase of the crossover frequency is lower than $-\pi$. This kind of crossing 


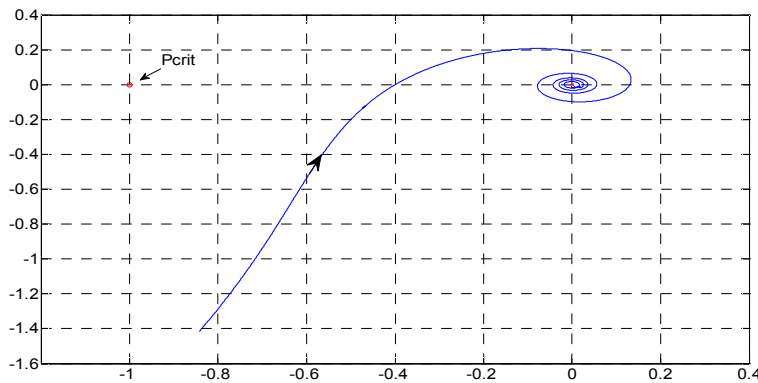

(a) L-type GCI.

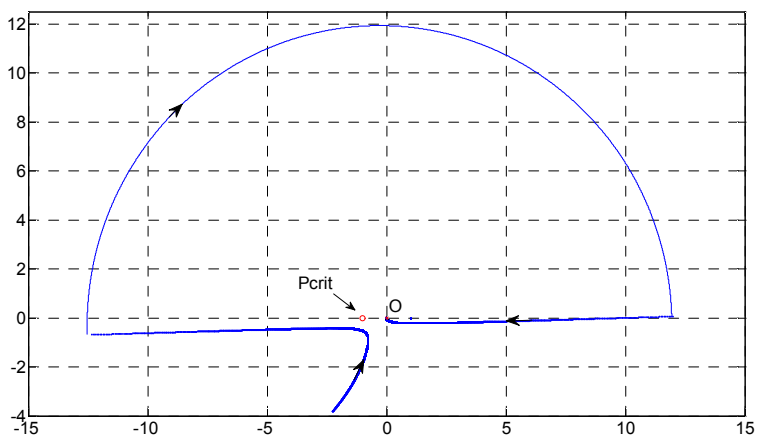

(b) LCL-type with $\omega_{r, p u}=1$.

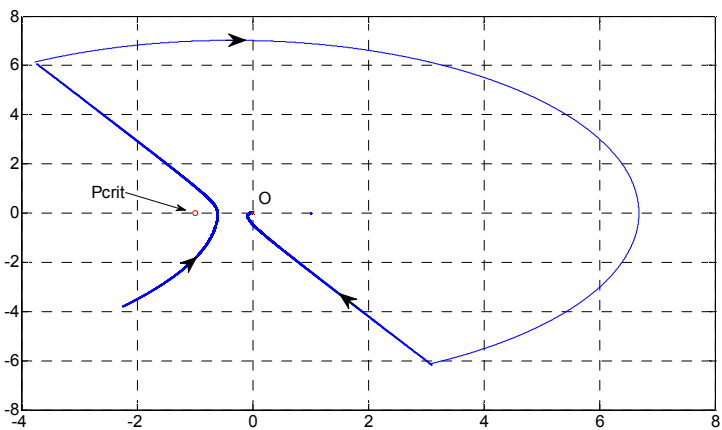

(c) LCL-type with $\omega_{r, p u}=1.74$.

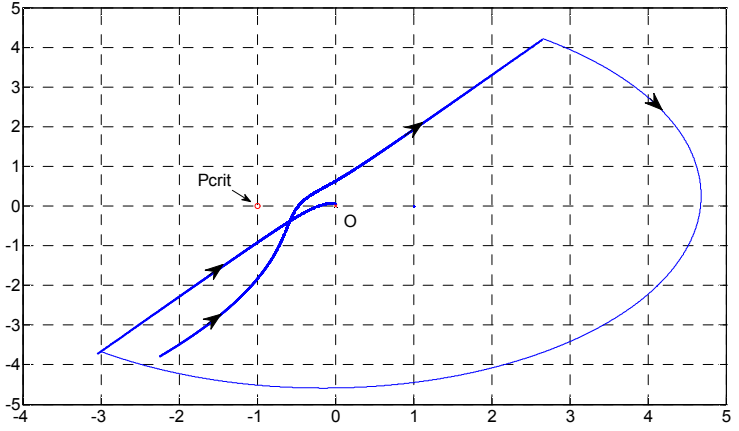

(d) LCL-type with $\omega_{r, p u}=2.5$.

Fig. 2. Open-loop Nyquist diagram of L-type and LCL-type GCI.

may lead to instability. Note that, as $\omega_{r, p u}$ increases, three crossover frequencies will appear. The phase angle of the lowest crossover frequency will become larger than $-\pi$, which could possibly ensure stability (see lines 2 and 3 ). This phenomenon can help design a controller more effectively, which means that the three crossover frequencies need to be identified and made to have enough phase margin by properly fixing the resonance frequency $\omega_{r}$. This can ensure the stability and performance of the system more accurately.

\section{DESIGN PROCEDURE}

The aim of this section is to present a systematic approach to select the main control parameters for the LCL-type GCIs, by taking the L-type control design as a first step.

\section{A. Identify the Crossover Frequencies of an LCL Filter to Adjust the PI Controller}

$\omega_{r}$ is increased and three crossover frequencies are made to appear. According to the Nyquist Stability Criterion, it is possible to ensure the stability and performance of systems by making them with enough of a phase margin. Fig. 3 shows the three crossover frequencies of line 2 , named $\omega_{c 1}, \omega_{c 2}$, and $\omega_{c 3}$ in ascending frequency order. Their corresponding phase angles are $\varphi_{1}=-\pi+\phi_{m 1}, \varphi_{2}=-\pi-\phi_{m 2}$ and $\varphi_{3}=-3 \pi+\phi_{m 3}$, where $\phi_{m 1}, \phi_{m 2}$ and $\phi_{m 3}$ represent the phase margins [17]. In addition, the gain margin is measured at a phase angle of $-\pi$, and the corresponding frequency is defined as $\omega_{-\pi}$. In Fig. 3, the points ' $a$ ', 'b', and 'c' denote the phase margin for line 2 , while point ' $d$ ' denotes its gain margin, and point 'e' denotes the gain margin of line 3 .

When the capacitor $C_{f}$ is removed from the LCL filter in Fig. 1, it becomes an $\mathrm{L}$ filter with $L=L_{1}+L_{2}$. The PI current controller can be designed easily because a comparatively large phase margin can be reserved, which is set as $\phi_{m}=55^{\circ}$ here. The integral term of the PI controller reduces the phase margin, i.e. about $5^{\circ}$. Hence, for an L-type GCI with a sample interval of $\mathrm{T}=50 \mu \mathrm{s}$, the phase margin $\phi_{m}$ can be set as $49.1^{\circ}$ and the gain margin $\lambda_{L}$ can be set as $7.97 \mathrm{~dB}$. According to these constrains, the parameters of the PI controller can be optioned to get the maximum band width $\omega_{c(\max )}[3]$.

The capacitor $C_{f}$ then returns and subsequently the $\mathrm{L}$ filter changes back to an LCL filter. It can be determined by a numerical evaluation that if $\omega_{r}$ approaches infinity, the stability margin will be determined by the gain margin at frequency $\omega_{-\pi}$, which is the same as its L-type counterpart (see Fig. 3). Assuming a gain margin $\lambda_{L C L}$ for the LCL-type GCI (it should be smaller than its L-type counterpart for the sake of feasibility) and referring to (2), the lower limit of $\omega_{r_{-} \text {low }}$ can be determined by solving:

$$
\lambda_{L}-\lambda_{L C L}=20 \cdot \log _{10}\left(\frac{\omega_{r_{-} l o w}^{2}}{\omega_{r_{-} \text {low }}^{2}-\omega_{-\pi}^{2}}\right)
$$




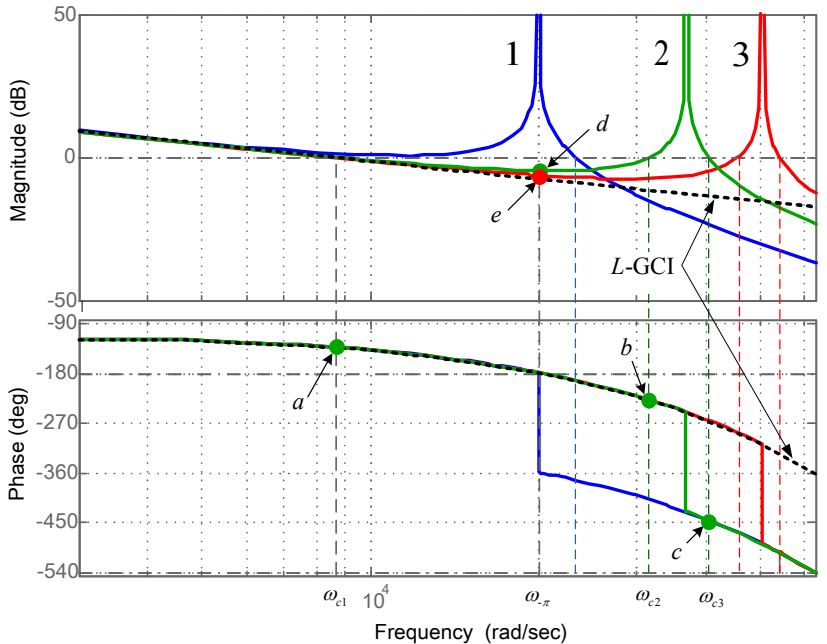

Fig. 3. Open-loop Bode diagram of LCL-type GCI against $\omega_{r, p u}$ with L-type counterpart (dashed line) as the benchmark.

(Line 1: $\omega_{r, p u}=1$; line 2: $\omega_{r, p u}=1.74$; line 3: $\omega_{r, p u}=2.5$ )

Although the gain margin increases with $\omega_{r}$, the phase margin $\phi_{m 3}$ decreases. Then from (2), the upper limit of $\omega_{r_{-} u p}$ can be found as follows:

$$
\left\{\begin{array}{l}
\frac{\omega_{c(\max )}}{\omega_{c 3}} \cdot \frac{\omega_{r_{-} u p}^{2}}{\omega_{r_{-} u p}^{2}-\omega_{c 3}^{2}} \approx-1 \\
\arctan \left(\frac{10 \omega_{c 3}}{\omega_{c(\max )}}\right)-\omega_{c 3} \mathrm{~T}_{\mathrm{d}}-2 \pi=-3 \pi+\phi_{m 3}
\end{array}\right.
$$

Although the stability is guaranteed, there is still a resonance peak, which may cause problems when the GCI is aiming to track current harmonics around the resonance frequency. However, in DG systems where GCIs are mostly in charge of energy conversion, the resonance peak should not be troublesome.

\section{B. Parameters Design of the LCL Filter}

Once the range of the resonance frequency $\omega_{r}$ has been identified, the parameters of the LCL filter should be determined. It is well known that the larger the total inductance of the filter, the larger the fundamental voltage drop. This also increases the amount of reactive power that can be absolved. On the other hand, for low values of the inductance, a high current ripple is obtained, which results in higher iron losses and more current stress on the semiconductors. Therefore, constraints on the inductances should be set as a priority. The preliminary parameters of the LCL filter, including the resonance frequency $\left(\omega_{r}\right)$, the maximum allowable grid current ripple in a percentage of the rated value $\left(\tilde{i}_{2}=\Delta i_{2} / I_{2, n}\right)$, and the inductor split factor ( $\left.k_{L}=L_{2} / L_{1}\right)$, are selected by using the following steps:
1) Design of $\tilde{i}_{2}$ : A lower value of $\tilde{i}_{2}$ requires a larger total required inductance. According to IEEE std. 519-1992 [28], the upper limit is $30 \%$ without a loss of generality. The value of $\tilde{i}_{2}$ can be initialized to $25 \%$ and then adjusted according to the amount of total inductance;

2) Design of $\omega_{r}$ : Given $\tilde{i}_{2}$, a larger value of the total inductance requires a higher $\omega_{r}$ [18]. Therefore, $\omega_{r}$ should be set as lower as possible, e.g. the lower limitation required by the PI controller;

3) Design of $k_{L}$ : The inverter current ripple reaches its maximum at $k_{L}=1$. Therefore, $k_{L}$ should be set as a trade-off between the current ripple (e.g., $\left.\tilde{i}_{1}=\Delta i_{1} / I_{2, n} \in[15 \%, 40 \%]\right)$ and the reactive power (e.g., $<5 \%$ ). A good trade-off can be achieved by selecting $k_{L}=0.3$.

After that, the values of the filter components can be determined. The harmonic in $u_{P W M}$ corresponding to the maximum carrier sideband harmonic of the grid current can be identified by applying a Bessel function. In terms of an H-bridge with a Unipolar SPWM, the $(2 N+1)^{\text {th }}$ order harmonic current ( $N$ is the carrier ratio) represents the most significant component [29]. The amplitude of the corresponding harmonic voltage is governed by:

$$
\hat{U}_{1,1}=\frac{2 U_{d c}}{\pi} J_{1}(\mathrm{M} \pi)
$$

here, the subscript of $\hat{U}_{1,1}$ represents the first sideband of the first carrier band, and $J_{1}$ is a first order Bessel function. M is the modulation depth. $\mathrm{M}$ varies little with load changes. Therefore, it can be approximated by the modulation depth for the no-load condition, i.e. $\mathrm{M}=\hat{u}_{g} / U_{d c}$, where $\hat{u}_{g}$ is the magnitude of $u_{g}$.

Back to Fig. 1, the output current can be determined as follows:

$$
i_{2}(s)=\frac{\omega_{r}^{2}}{s\left(L_{1}+L_{2}\right)\left(s^{2}+\omega_{r}^{2}\right)}\left[u_{P W M}(s)-\left(L_{1} C_{f} s^{2}+1\right) \cdot u_{g}(s)\right]
$$

When $u_{P W M}(s)=\hat{U}_{1,1}$ and $u_{g}(s)=0$, the inverter-side inductance can be set as:

$$
L_{1}=\frac{\hat{U}_{1,1}}{\sqrt{2} \cdot \tilde{i}_{2} I_{2, n}\left(1+k_{L}\right) \omega_{2 N+1}} \cdot \frac{\omega_{r}^{2}}{\omega_{2 N+1}^{2}-\omega_{r}^{2}}
$$

where $\omega_{2 N+1}$ is the angular frequency of the $(2 N+1)^{\text {th }}$ order harmonic.

The grid-side inductance and filter capacitance can be selected as:

$$
L_{2}=k_{L} L_{1}
$$


TABLE I

PARAMETERS OF LCL Filter AND REACTIVE COMPONENT

\begin{tabular}{|c|c|}
\hline$U_{d c}$ & $378 \mathrm{~V}$ \\
\hline$u_{g}$ & $220 \mathrm{~V}_{\text {rms }}$ \\
\hline$I_{2, n}$ & $11.5 \mathrm{~A}_{\mathrm{rms}}$ \\
\hline$L_{1}$ & $1.2 \mathrm{mH}$ \\
\hline$L_{2}$ & $0.35 \mathrm{mH}$ \\
\hline$C_{f}$ & $3.3 \mu \mathrm{F}$ \\
\hline$\omega_{r, p u}$ & 1.64 \\
\hline$\tilde{i}_{2}$ & $25 \%$ \\
\hline
\end{tabular}

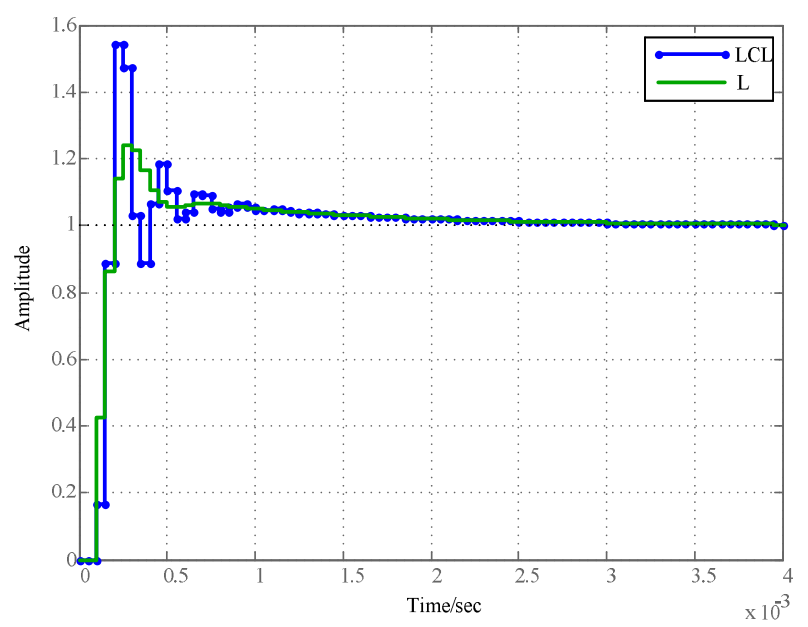

Fig. 4. Step responses of L-GCI and LCL-GCI.

and:

$$
C_{f}=\frac{L_{1}+L_{2}}{L_{1} L_{2} \omega_{r}^{2}}
$$

\section{DESIGN EXAMPLE WITH PERFORMANCE EVALUATION}

According to the above procedure, a design example of a $2.5 \mathrm{~kW}$ GCI with an LCL filter is presented in Table I, where the total inductance is 0.025 p.u., the inverter current ripple is $25 \%$, and the capacitive power is 0.02 p.u. Consequently, the presented design fits well with the practical constraints. Then, the evaluation of control performance is carried out as follows.

\section{A. Reference Tracking}

The load step response of both the L-type and the LCL-type filters are presented in Fig. 4. They show a similar bandwidth but a higher overshoot that lasts for about $2 \mathrm{~T}$. However, such a peak only occurs in the averaged model. Hence, it can be used to check the stability margins. During the initial overshoot, the averaged model is not valid. As a result, a more smooth dynamics is available, as proved in Section V.

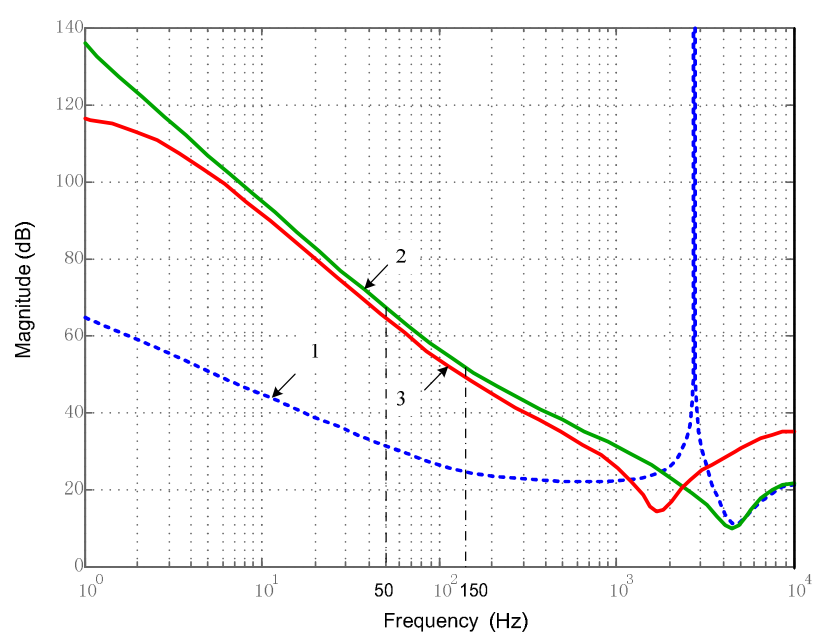

Fig. 5. Output impedance of LCL-GCI.

(Line 1: $L_{g}=0, G_{f f}(s)=0 ;$ line $2: L_{g}=0, G_{f f}(s)=1 ;$ line 3 : $\left.L_{g}=3 L_{2}, \quad G_{f f}(s)=1\right)$.

\section{B. Rejection of Grid Disturbances}

The inverter output impedance is defined as:

$$
Z_{o}(s)=u_{g}(s) / i_{2}(s)
$$

The larger the value of $Z_{o}$, the stronger the rejection of grid voltage disturbances [10] [26]. Hence, if the output impedance is high enough in the whole frequency range, the power quality will be much more improved in terms of harmonic distortion. Fig. 1(b) shows that if an exact full grid voltage feed-forward control is employed:

$$
G_{f f}(s)=\left(L_{1} C_{f} s^{2}+1\right) \cdot e^{s \mathrm{~T}_{\mathrm{d}}}
$$

The output impedance tends to be infinite. However, the second-order differentiator and the lead phase blocks can be neglected. Thus, it is possible to approximate $e^{s \mathrm{~T}_{\mathrm{d}}} \approx 1$ and $L_{1} C_{f} s^{2} \ll 1$, so that $G_{f f}(s)=1$ is still preferred. With these assumptions, the Bode diagram is illustrated in Fig. 5. It shows a lot of improvement in the output impedance (compare line 2 and line 1), which is only little degraded by the grid impedance. It is worth mentioning that if multi-feedback active damping control is employed (e.g., capacitor current feedback [27]), the subsequent negative impact from the grid impedance will be noticeable. As a result, the traditional unity feed-forward control $G_{f f}(s)=1$ may not be the best solution.

\section{Inherent Damping Characteristic from the Feed-Forward Control of Grid Voltage}

When unity grid voltage feed-forward control is used, the grid voltage $u_{g}$ is measured at the point of common coupling (PCC). Assuming weak grid conditions, i.e., $L_{g}=n L_{2}$, the grid voltage can be expressed as follows: 


$$
u_{g}=\frac{n}{1+n} u_{C f}+\frac{1}{1+n} u_{S}
$$

The open-loop transfer function of an LCL-type GCI with the grid voltage feed-forward control can be expressed as:

$$
G_{L C L, g}^{o}(s)=G_{L, g}^{o}(s) \cdot \frac{\omega_{r, g}^{2}}{s^{2}+\omega_{r, g}^{2}\left[1-G_{f f}(s) \cdot \frac{n k_{L}}{1+(1+n) k_{L}} e^{-s \mathrm{~T}_{\mathrm{d}}}\right]}
$$

where $\omega_{r, g}=\sqrt{\frac{L_{1}+L_{2}+L_{g}}{L_{1}\left(L_{2}+L_{g}\right) C}}$.

While the open-loop transfer function of the corresponding L-type counterpart can be expressed as:

$$
G_{L, g}^{o}(s)=\frac{U_{d c} \cdot G_{P I}(s) \cdot e^{-s \mathrm{~T}_{\mathrm{d}}}}{L_{T, g} s}
$$

where $L_{T, g}=L_{1}+L_{2}+L_{g}$.

The delay block $e^{-s \mathrm{~T}_{\mathrm{d}}}$ can be approximated by its first order Taylor series, i.e. $e^{-s \mathrm{~T}_{\mathrm{d}}} \approx 1-\mathrm{T}_{\mathrm{d}} \cdot s$. Hence, (13) can be expressed as:

$$
G_{L C L, g}^{o} \approx G_{L C L, g}^{o} \frac{\omega_{r, g}{ }^{2}}{s^{2}+K_{D} \mathrm{~T}_{\mathrm{d}} \omega_{r, g}{ }^{2} s+\left(1-K_{D}\right) \cdot \omega_{r, g}{ }^{2}}
$$

where $K_{D}=g_{f f} \frac{n k_{L}}{1+(1+n) k_{L}} \in(0,1)$.

From (15), it can be observed that if $G_{f f}(s)$ is a positive constant $g_{f f}$, a damping coefficient $\zeta$ is introduced due to the phase delay introduced by $e^{-s \mathrm{~T}_{\mathrm{d}}}$. This can be expressed as:

$$
\zeta=\frac{1}{2} \frac{K_{D}}{\sqrt{1-K_{D}}} \omega_{r, g} \mathrm{~T}_{\mathrm{d}}
$$

As illustrated in Fig. 6, the unity feed-forward under a weak grid not only preserves the stability margin, but also makes the loop gain almost unchanged (compare line 3 with line 2). Compared with the existing active damping methods, where the bandwidth is mostly reduced by negative feedback of state variables, the proposed approach gives the active damping benefit without any additional control loop.

\section{SIMULATION AND EXPERIMENTAL RESULTS}

\section{A. Simulation Results}

A Matlab/Simulink model is developed to verify the proposed integral design of the GCI together with the LCL filter, using the parameters listed in Table I. The harmonics content shown in Table II are introduced to the grid voltage. Load step changes from no-load to full-load and vice versa are performed under weak grid condition with different grid impedances.

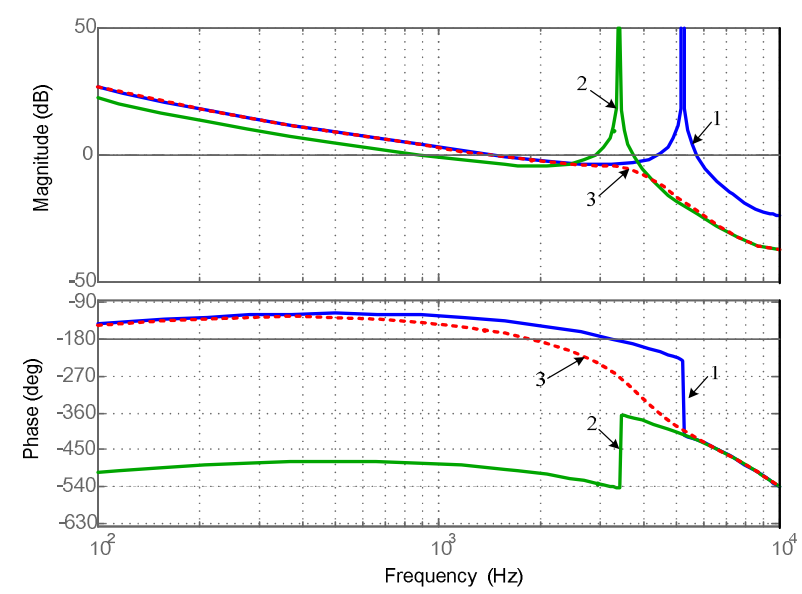

Fig. 6. Open-loop Bode diagram against grid impedance.

(1. $L_{g}=0, G_{f f}(s)=0 ; 2 . L_{g}=3 L_{2}, G_{f f}(s)=0 ; 3$. $L_{g}=3 L_{2}$, $\left.G_{f f}(s)=1\right)$.

TABLE II

COMPONENTS OF GRID HARMONICS

\begin{tabular}{|l|l|}
\hline $3^{\text {rd }}$ & $4.82 \%$ \\
\hline $5^{\text {th }}$ & $4.18 \%$ \\
\hline $7^{\text {th }}$ & $3.54 \%$ \\
\hline $9^{\text {th }}$ & $2.9 \%$ \\
\hline
\end{tabular}

The waveforms shown in Fig. 7(a) illustrate that by using a PI controller, the grid current is severely distorted, and the compatibility of the grid impedance is poor, due to the resonance at the load switching instant if $L_{g}=L_{2}$. However, such a condition is greatly improved by the grid voltage feed-forward control, as shown in Fig. 7(b), where $L_{g}=3 L_{2}$. Moreover, the system dynamics are smoother than the averaging model based analysis (see Fig. 4, which has stiff grid conditions), due to the strong proportional part and the comparatively weak integral part of the PI controller. Actually, the LCL-type GCI behaves similar to the L-type one, except for the sharp harmonic attenuation, as detailed in Fig. 7(c), where the switching grid current under stiff grid conditions is about $0.26 \%$ of the rated current.

\section{B. Experimental Results}

The proposed design strategy is experimentally verified on a $2.5 \mathrm{~kW}$ prototype, as shown in Fig. 8 .

The dc and ac buses are formed by an isolated dc source and the utility grid, respectively. The control is implemented by a 32 bit fixed-point $100 \mathrm{MHz}$ TMS320F2808 DSP. Unipolar-SPWM is used, the switching frequency is $10 \mathrm{kHz}$ and the sampling frequency is $20 \mathrm{kHz}$. Since there is no need for active damping, only the grid current and the grid voltage are sensed. The sampling action is taken at the top and bottom of the carrier signal. The dead time for the power switches is set to $1 \mu \mathrm{s}$. The parameters are selected as shown 


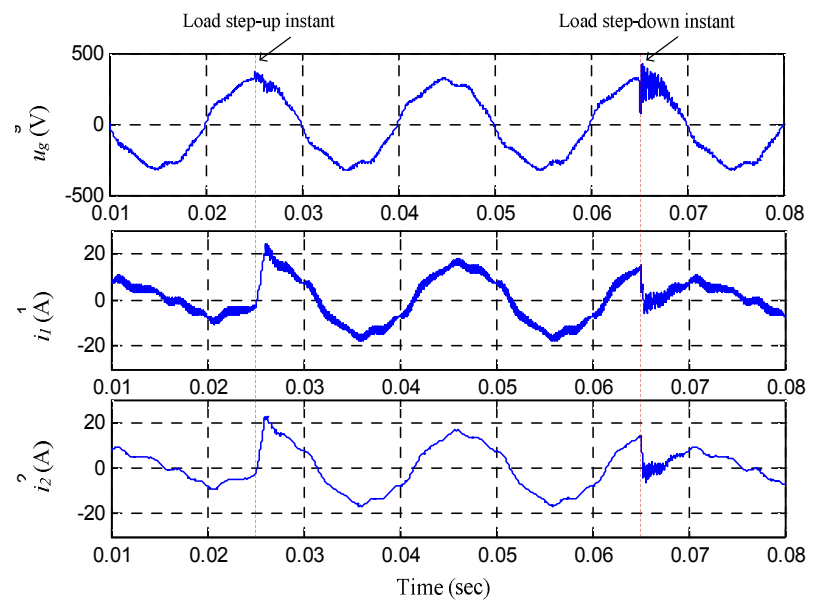

(a) $G_{f f}(s)=0, \quad L_{g}=L_{2}$
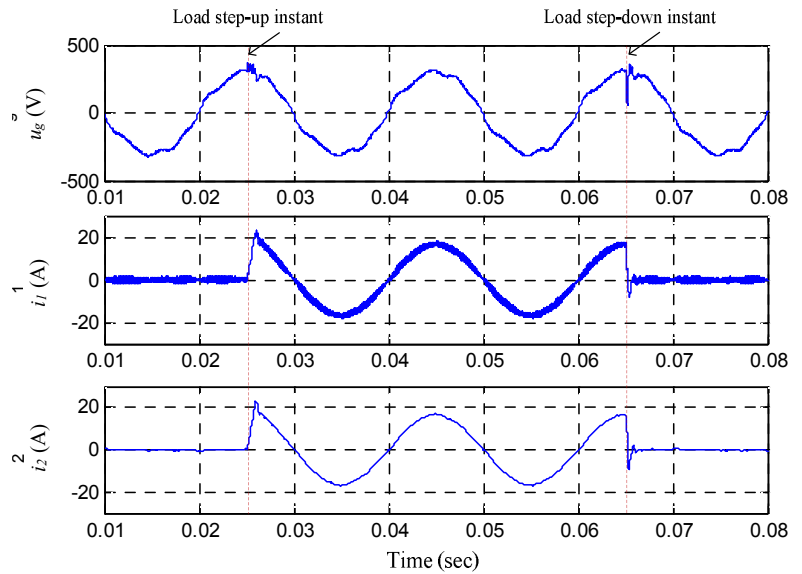

(b) $G_{f f}(s)=1, \quad L_{g}=3 L_{2}$

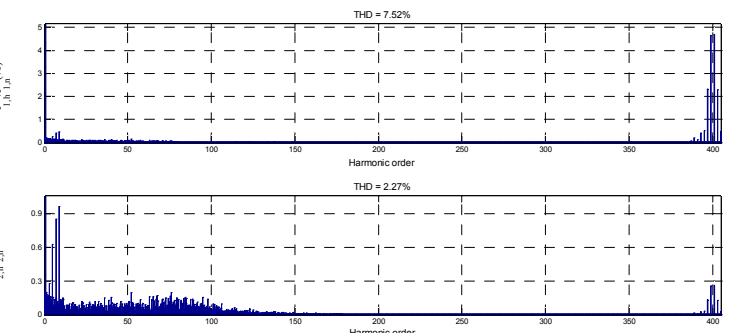

(c) Harmonic spectrum. (Subscripts $h$ and $n$ denote harmonics and fundamental components respectively.)

Fig. 7. Simulations under weak and stiff grid conditions.

\section{in Table I.}

Firstly, under stiff grid conditions, load steps from no-load to full-load, and vice versa, are performed. The resulting waveforms of the inverter side current and grid side current are shown in Fig. 9. They show smooth and fast dynamics and good power quality. The THD of the grid current is well below the standard requirements, as shown in Fig. 9(b) (i.e. $2.78 \%$ vs. $5 \%$ ). Notice that there is some deviation that is mostly accounted for by the measurement susceptible to EMI during IGBT switching, and reflected by the harmonics of the $200^{\text {th }}$ order that should theoretically be null. Note that $\tilde{i}_{2}$ is

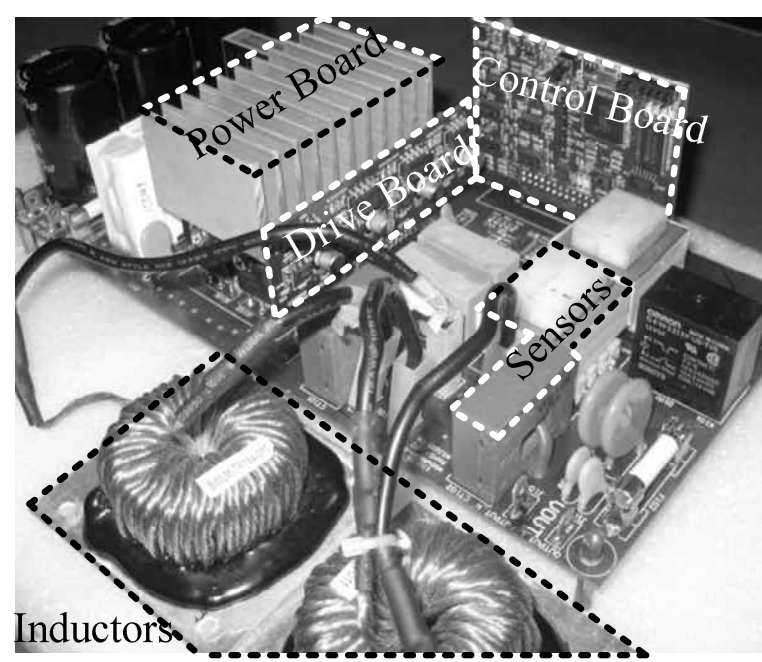

Fig. 8. Photograph of the single phase GCI prototype.

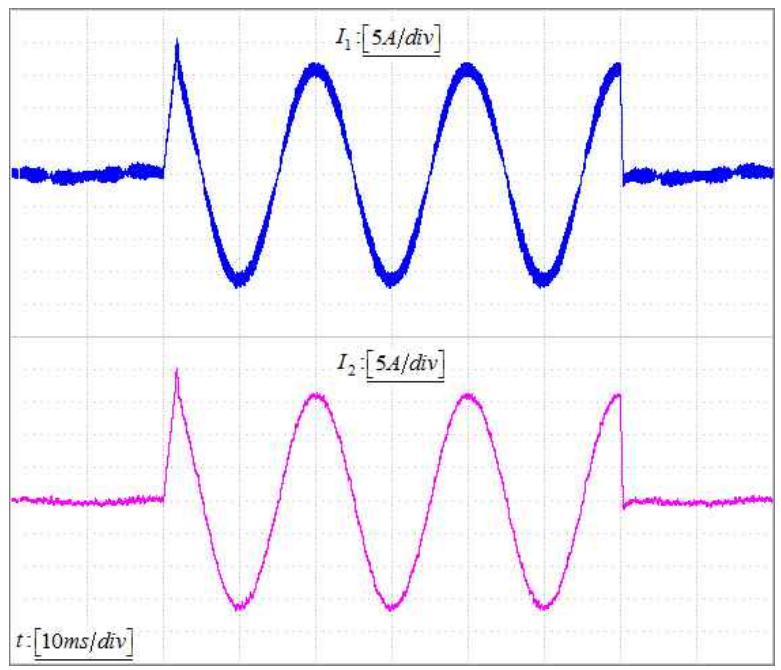

(a) $G_{f f}(s)=0, \quad L_{g}=L_{2}$
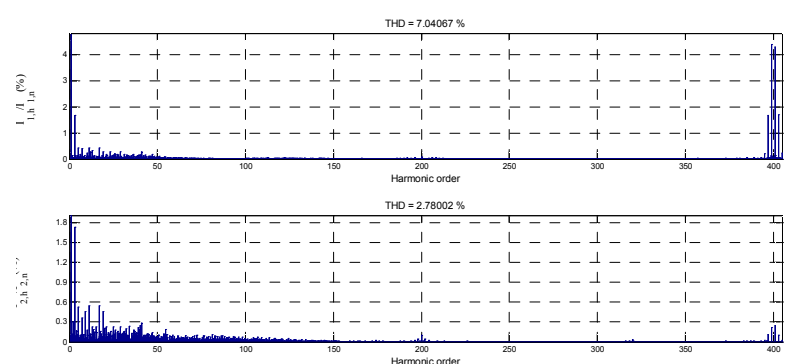

(b) $G_{f f}(s)=1, \quad L_{g}=3 L_{2}$

Fig. 9. Simulations under weak and stiff grid conditions.

about $25 \%$, implying that the dominant iron loss is low.

Then, load steps under weak grid conditions are emulated by inserting another inductor $L_{g}=1 \mathrm{mH}$ between the PCC and the grid. The waveforms shown in Fig. 10 illustrate the good behavior of the system even for large grid impedance values. Moreover, there is no obvious deterioration of the power quality. This is ensured by the large output impedance 


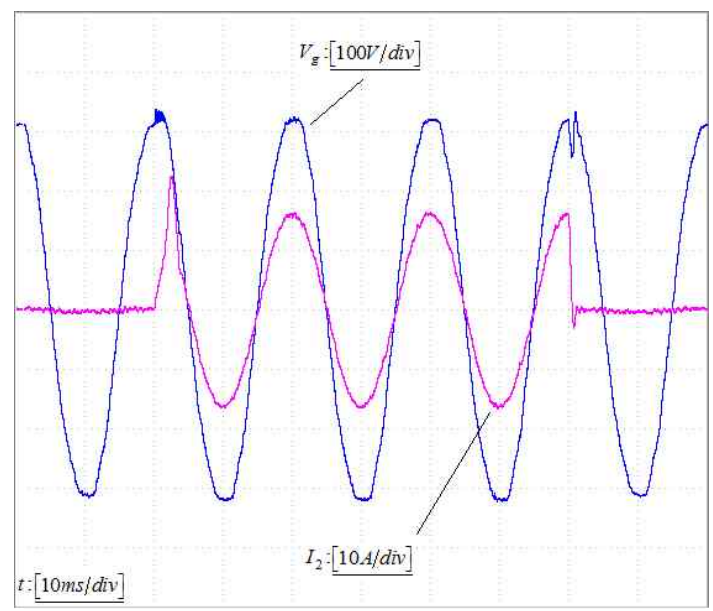

Fig. 10. Experimental waveforms of grid voltage and current in weak grid $\left(L_{\mathrm{g}}=1 \mathrm{mH}\right)$.

and the preserved loop gain (see Fig. 5 and Fig. 6).

\section{CONCLUSIONS}

The integral design of a grid-connected inverter (GCI) control and its LCL filter is proposed in this paper. First, the capacitor is removed from the LCL filer and the design starts from a GCI with an L filter and a PI controller. Then, the capacitor returns to the filter and introduces a resonance, which is identified to accommodate the preset PI controller by identifying the phase margin of three cross over frequencies. Furthermore, the performance under distorted and weak grid conditions is ensured by the unity feed-forward of the grid voltage. Moreover, the feed-forward control of the grid voltage results in an inherent damping effect on the resonance. Hence, there is no need for any kind of active damping control. In such an easy way, the controller and filter design are set as a whole system, so that the control merits of the L-type GCI and the harmonic attenuation merits offered by the LCL filter are well integrated. The good dynamics, power quality, and strong robustness in terms of grid impedance variations are verified by simulation and experimental results. This shows that the proposed design is a promising tool for distributed generation systems.

\section{REFERENCES}

[1] M. Liserre, F. Blaabjerg, and S. Hansen, "Design and control of an LCL-filter-based three-phase active rectifier," IEEE Trans. Ind. Applicat., Vol. 41, No. 5, pp. 1281-1291, Sep./Oct. 2005.

[2] T. C. Wang, Z. Ye, G. Sinha, and X. Yuan, "Output filter design for a grid-interconnected three-phase inverter," in Power Electronics Specialist Conference (PESC), Vol. 2, pp. 779-784, 2003.

[3] D. G. Holmes, T. A. Lipo, B. P. McGrath, and W. Y. Kong, "Optimized design of stationary frame three phase ac current regulators," IEEE Trans. Power Electron., Vol. 24, No. 11, pp. 2417-2426, Nov. 2009.

[4] J. Dannehl, M. Liserre, and F. W. Fuchs, "Filter-based active damping of voltage source converters with LCL filter," IEEE Trans. Ind. Electron., Vol. 58, No. 8, pp. 3623-3633, Aug. 2011.

[5] J. Dannehl, F. W. Fuchs, and P. B. Thogersen, "PI state space current control of grid-connected PWM converters with LCL filters," IEEE Trans. Power Electron., Vol. 25, No. 9, pp. 2320-2330, Sep. 2010.

[6] M. Xue, Y. Zhang, Y. Kang, Y. Yi, S. Li, and F. Liu, "Full feedforward of grid voltage for discrete state feedback controlled grid-connected inverter with LCL filter," IEEE Trans. Power Electron., Vol. 27, No. 10, pp. 4234-4247, Oct. 2012.

[7] S. G. Parker, B. P. McGrath, and D. G. Holmes. "Regions of active damping control for LCL filters," in Energy Conversion Congress and Exposition (ECCE'12), pp. 53-60, 2012.

[8] M. Liserre A. D. Aquila, and F. Blaabjerg, "Stability improvements of an LCL-filter based three-phase active rectifier," in Power Electronics Specialists Conference (PESC), Vol. 3, pp. 1195-1201, 2002.

[9] M. Liserre, A. D. Aquila, and F. Blaabjerg, "Genetic algorithm-based design of the active damping for an LCL-filter three-phase active rectifier," IEEE Trans. Power Electron., Vol. 19, No. 1, pp. 76-86, Jan. 2004.

[10] E. Twining and D. G. Holmes, "Grid current regulation of a three-phase voltage source inverter with an LCL input filter," IEEE Trans. Power Electron., Vol. 18, No. 3, pp. 888-895, May 2003.

[11] I. J. Gabe, V. I. F. Montagner, and H. Pinheiro, "Design and implementation of a robust current controller for VSI connected to the grid through an LCL filter," IEEE Trans. Power Electron., Vol. 24, No. 6, pp. 1444-1452, Jun. 2009.

[12] E. Wu and P. W. Lehn, "Digital current control of a voltage source converter with active damping of LCL resonance," IEEE Trans. Power Electron., Vol. 21, No. 5, pp. 1364-1373, Sep. 2006.

[13] G. Shen, D. Xu, L. Cao, and X. Zhu, "An improved control strategy for grid-connected voltage source inverters with an LCL filter," IEEE Trans. Power Electron., Vol. 23, No. 4, pp. 1899-1906, July 2008.

[14] G. Shen, X. Zhu, J. Zhang, and D. Xu, "A new feedback method for PR current control of LCL-filter-based grid-connected inverter," IEEE Trans. Ind. Electron., Vol. 57, No. 6, pp. 2033-2041, Jun. 2010.

[15] X. Bao, F. Zhuo, Y. Tian, and P. Tan, "Simplified feedback linearization control of three-phase photovoltaic inverter with an LCL filter," IEEE Trans. Power Electron., Vol. 28, No. 6, pp. 2739-2752, Jun. 2013.

[16] Y. Tang, P. C. Loh, P. Wang, F. H. Choo, and F. Gao, "Exploring inherent damping characteristic of LCL-filters for three-phase grid-connected voltage source inverters," IEEE Trans. Power Electron., Vol. 27, No. 3, pp. 1433-1443, Mar. 2012.

[17] M. Driels, Linear Control Systems Engineering, McGraw-Hill, 1996.

[18] K. Jalili and S. Bernet, "Design of LCL filters of active-front-end two-level voltage-source converters," IEEE Trans. Ind. Electron., Vol. 56, No. 5, pp. 1674-1689, May 2009.

[19] A. A. Rockhill, M. Liserre, R. Teodorescu, and P. Rodriguez, "Grid-filter design for a multi-megawatt medium-voltage voltage-source inverter," IEEE Trans. Ind. Electron., Vol. 58, No. 4, pp. 1205-1217, Apr. 2011.

[20] M. Castilla, J. Miret, J. Matas, L. Garcia de Vicuna, and J.M Guerrero, "Control design guidelines for single-phase grid-connected photovoltaic inverters with damped resonant 
harmonic compensators," IEEE Trans. Ind. Electron., Vol. 56, No. 11, pp. 4492-4501, Nov. 2009.

[21] A. A. Luiz and B. J. C. Filho, "Minimum reactive power filter design for high power three-level converters," in Annual Conference of IEEE Industrial Electronics Society (IECON), pp. 3272-3277, 2008.

[22] J. M. Uhlethaler, M. Schweizer, R. Blattmann, J. W. Kolar, and A. Ecklebe, "Optimal design of LCL harmonic filters for three-phase PFC rectifiers," in Annual Conference of IEEE Industrial Electronics Society (IECON), pp. 1438-1445, Nov. 2011

[23] P. Channegowda and V. John, "Filter optimization for grid interactive voltage source inverters," IEEE Trans. Ind. Electron., Vol. 57, No. 12, pp. 4106-4114, Dec. 2010.

[24] Y. Tang, P. C. Loh, P. Wang, F. H. Choo, F. Gao, and F. Blaabjerg, "Generalized design of high performance shunt active power filter with output LCL filter," IEEE Trans. Ind. Electron., Vol. 59, No. 3, pp. 1443-1452, Mar. 2012.

[25] J. Kim, J. Choi, and H. Hong, "Output LC filter design of voltage source inverter considering the performance of controller," in International Conference on Power System Technology (PowerCon), Vol. 3, pp. 1659-1664, 2000.

[26] T. Abeyasekera, C. M. Johnson, D. J. Atkinson, and M. Armstrong, "Suppression of line voltage related distortion in current controlled grid connected inverters," IEEE Trans. Power Electron., Vol. 20, No. 6, pp. 1393-1401, Nov. 2005.

[27] X. Wang, X. Ruan, S. Liu, and C. K. Tse, "Full feedforward of grid voltage for grid-connected inverter with LCL filter to suppress current distortion due to grid voltage harmonics," IEEE Trans. Power Electron., Vol. 25, No. 12, pp. 3119-3126, Dec. 2010.

[28] IEEE Recommended Practices and Requirements for Harmonic Control in Electrical Power Systems, IEEE Std. 519-1992, 1992.

[29] D. G. Holmes and T. A. Lipo, Pulse Width Modulation for Power Converters, Wiley, 2003.

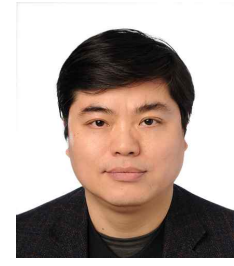

Yu Zhang was born in Jiangsu Province, China. He received his B.E., M.E. and Ph.D. degrees in Electrical Engineering from the Huazhong University of Science and Technology (HUST), Wuhan, China, in 1992, 1995 and 2005, respectively. From 1995 to 2002, he was an Engineer working on power supply applications in a telecommunication company. He is currently an Associate Professor in the School of Electrical and Electronic Engineering, HUST, where he teaches power electronics. His current research interests include power electronics modeling and control, parallel UPSs, and renewable energy generation. He has developed several power systems, such as UPSs, and achieved 4 Scientific and Technological Progress Awards from the Chinese government and various academic societies. He is the author of more than 60 technical papers and is now a Member of the UPS Standard Committee of China.

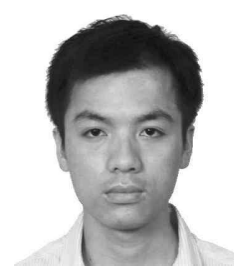

Mingyu Xue was born in Zhejiang Province, China, in 1984. He received his B.S. degree in Electrical Engineering and Automation from the Dalian University of Technology (DLUT), Dalian, China, in 2006, and his M.S degree in Power Electronics and Drives from the Huazhong University of Science and Technology (HUST), Wuhan, China, in 2008 He is currently working toward his Ph.D. degree at HUST. He is now with the Shanghai United Imaging Healthcare Company.
His current research interests include power electronic converters, renewable energy and distributed generation.

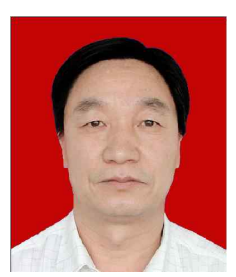

Minying Li was born in Shanxi Province, China. He received his B.E. degree in Electronics Engineering from the Shanxi Technology College, Xian, China, in 1983. He joint the Guangdong Zhicheng Champion Group Company, in 1999, and is currently the Chief Engineer of this company. He has developed many types of power systems, such as UPSs. He received a National Golden Medal of Patent in 2005 and was awarded as a Distinguish Specialist with a special allowance from the State Council of China. He is now the secretary general of the UPS Standard Committee of China.

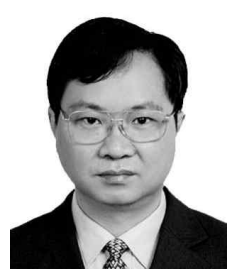

Yong Kang was born in Hubei Province, China. He received his B.E., M.E., and Ph.D. degrees from the Huazhong University of Science and Technology (HUST), Wuhan, China, in 1988, 1991 and 1994, respectively. In 1994, he joined HUST as a Lecturer and was promoted to Associate Professor, in 1996, and to Full Professor, in 1998. He is currently the Vice Chairman of the China UPS Standard Committee and the Head of the School of Electrical and Electronic Engineering, HUST. His current research interests include power electronic converters, ac drivers, renewable energy generation, and EMC techniques. He is the author of more than 200 technical papers and is an associate editor of the Journal of Power Electronics.

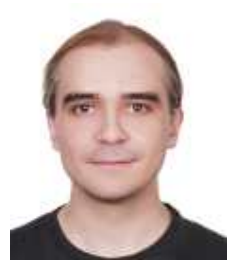

Josep M. Guerrero received his B.S. degree in Telecommunications Engineering, his M.S degree in Electronics Engineering, and his Ph.D. degree in Power Electronics from the Technical University of Catalonia, Barcelona, Spain, in 1997, 2000 and 2003, respectively. Since 2011, he has been a Full Professor with the Department of Energy Technology, Aalborg University, Denmark, where he is responsible for the Microgrid Research Program. Since 2012, he has been a Guest Professor at the Chinese Academy of Science, Beijing, China, and the Nanjing University of Aeronautics and Astronautics, Nanjing, China. Since 2014, he has been a Chair Professor at Shandong University, Jinan, China. His current research interests include different microgrid aspects, including power electronics, distributed energy-storage systems, hierarchical and cooperative control, energy management systems, and the optimization of microgrids and islanded minigrids. Professor Guerrero is an Associate Editor for the IEEE Transactions on Power Electronics, the IEEE Transactions on Industrial Electronics, and the IEEE Industrial Electronics Magazine. He is also an Editor for the IEEE Transactions on Smart Grid. He has been a Guest Editor of the IEEE Transactions on Power Electronics Special Issues: Power Electronics for Wind Energy Conversion and Power Electronics for Microgrids; the IEEE Transactions on Industrial Electronics Special Sections: Uninterruptible Power Supplies systems, Renewable Energy Systems, Distributed Generation and Microgrids, and Industrial Applications and Implementation Issues of the Kalman Filter; and the IEEE Transactions on Smart Grid Special Issue on Smart DC Distribution Systems. He was the Chair of the Renewable Energy Systems Technical Committee of the IEEE Industrial Electronics Society. In 2014, he was awarded as an ISI Highly Cited Researcher. 